\title{
Multiportal Combined Transorbital and Transnasal Endoscopic Resection of Fibrous Dysplasia
}

\author{
Tristan Tham ${ }^{1}$ Peter Costantino ${ }^{1}$ Margherita Bruni ${ }^{1}$ \\ laryngology, New York Head \& Neck Institute, \\ ${ }^{1}$ Department of Otolaryngology, New York Head \& Neck
Lenox Hill Hospital, New York, New York, United States \\ 2 Department of Neurosurgery, New York Head \& Neck Institute, Lenox \\ Hill Hospital, New York, New York, United States
}

\author{
David Langer $^{2}$ John Boockvar ${ }^{2}$ Prabhjyot Singh ${ }^{1}$
}

J Neurol Surg Rep 2015;76:e291-e296.

\begin{abstract}
Keywords

- combined multiportal endoscopic skull base surgery

- transnasal

- transorbital

Introduction Historically, access to the anterior skull base was achieved with open procedures. The paradigms to this approach were challenged with the advent of minimally disruptive endoscopic surgical techniques and supporting technology. The next step in the evolution of minimally disruptive surgery was the combination of multiportal endoscopic surgery.

Results The patient was an 18-year-old man who presented with right-sided proptosis. Further diagnostic tests revealed a fibrous dysplasia (FD) occupying the skull base and orbit. The lesion was successfully resected.

Conclusions The location of the tumor in this case was challenging, in which surgeons at some centers would have opted to have performed as an open procedure instead of endoscopically. The combined transnasal/transorbital approach is an uncommonly used technique that we have used to remove this tumor successfully. The patient also had a unique disease (FD) in a unique location that was treated without complications. This case report highlights how surgeons may use an expanded armamentarium in dealing with complex pathologies.
\end{abstract}

\author{
Address for correspondence Tristan Tham, MD, Department of \\ Otolaryngology, Head \& Neck Surgery (Black Hall), Lenox Hill Hospital, \\ 130 East 77th Street, 10th Floor, New York, NY 10075 \\ (e-mail: ttham@nshs.edu).
}

\section{Introduction}

Historically, access to the anterior skull base was achieved with open procedures. ${ }^{1}$ The paradigms to this traditional approach were challenged with the advent of minimally disruptive endoscopic surgical techniques and supporting technology. These newer surgical techniques offered a reduction in postoperative scarring, morbidity, and hospital length of stay as compared with traditional open surgeries. ${ }^{2}$ Minimally disruptive approaches to the anterior skull base and inferior portions of the anterior and middle cranial fossa are now widely practiced, where previously open craniofacial resections with higher rates of morbidity were the norm.

received

June 9, 2015

accepted after revision

August 19, 2015

published online

October 25, 2015
Endoscopic transnasal approaches to the anterior skull base are now commonly used, with easy access to the sella, suprasellar, and clivus regions. ${ }^{3,4}$ However, the major limitation is the angles of access available through the nose only. Surgeons operating via transnasal portals may have difficulty dealing with pathologies at or near the optic nerves and chiasm, infundibulum, hypothalamic vessels, and the anterior cranial arteries. ${ }^{5}$ Furthermore, transnasal surgery takes place in a nonsterile environment, and due to the curvature of the nose, endoscopes used in transnasal endoscopy often are angled, which can be visually disorienting for some surgeons. Extensive reconstructive procedures are also needed to prevent cerebrospinal fluid (CSF) leak postoperatively as well as follow-up intranasal debridements.
License terms

Stuttgart · New York

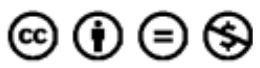


Transorbital approaches to access the anterior cranial fossa are increasingly described in the literature. ${ }^{6}$ This transorbital approach allows surgeons to overcome some of the limitations of the more historically relevant transnasal endoscopic approach, chiefly by an increased availability of angles of approach to the target pathology. Access to the ventral portion of the anterior cranial fossa outside of the central corridor is problematic in the transnasal approach because instruments have to traverse over critical neurovascular structures that lead into the orbit. ${ }^{7}$ In the transorbital approach, the orbital contents including the optic nerve can be seen and safely avoided. ${ }^{6}$

The other advantage of the transorbital approach includes the fact that the orbits are spaced more widely than the nares, allowing more workspace for multiple instruments and hands. The intended surgical target regions within the anterior fossa can often be approached in a coplanar manner transorbitally, thereby requiring fewer angled endoscopes, decreasing disorientation. ${ }^{6}$ Four different transorbital approaches-medial, lateral, superior, and inferior-could provide entry into the anterior fossa from many different directions that would allow the combination of multiple procedures into one surgery. ${ }^{6}$

Transorbital approaches have been used to repair traumatic CSF leaks, perform optic nerve decompression, and resect a variety of tumors including frontal and skull base mucoceles and mucopyoceles. ${ }^{8}$ Other tumors that have been resected successfully using a transorbital approach include adenoid cystic carcinoma of the skull base and orbits, as well as metastatic squamous cell carcinoma in the infraorbital nerve. ${ }^{6}$ Other pathologies include orbital and intracranial abscesses, control of epistaxis, orbital decompression, as well as decompression of the frontal sinus. ${ }^{8}$ Transorbital surgery can also be used to perform endoscopic craniotomy, in place of a full craniotomy. ${ }^{9}$

More novel uses of transorbital approaches include a modified form of transorbital endoscopic amygdalohippocampectomy (TEA) to perform hippocampus resection in patients with medically intractable epilepsy. ${ }^{9}$ Transorbital endoscopic surgery has been used to resect tumors in all areas of the anterior cranial fossa including the ethmoid and sphenoid sinuses.

The safety of transorbital endoscopic surgery has also been well described. In a large case series of 20 patients who underwent transorbital neuroendoscopic surgery for a wide variety of pathologies, no serious complications were noted, except a single case of mild enophthalmos. ${ }^{6}$

The next step in the evolution of minimally disruptive surgery of the anterior skull base was the combination of multiportal endoscopic surgery. By having the option of both transorbital and transnasal procedures at their disposal, surgeons have an expanded armamentarium in which to deal with complex or hard-to-reach pathologies that may otherwise have been difficult to access using traditional single-portal methods.

In this article we present a case report in which we have used both transnasal and transorbital techniques to complement each other in resection of a fibrous dysplasia (FD), which we believe is a novel situation. A review of the relevant literature is also performed.

\section{Setting}

The New York Head and Neck Institute (NYHNI) is a fullservice otolaryngology department at Lenox Hill Hospital, part of the North Shore Long Island Jewish Health System. The NYHNI serves a diverse patient population with a wide range of head and neck diseases in a tertiary hospital setting.

\section{Case Description}

The patient was an 18-year old man who presented to our clinic with a history of facial asymmetry of the right orbital region. He denied any symptoms other than the unilateral cosmetic change. He had no diplopia, blurry vision, loss of visual fields, or any other visual changes. No headaches or signs of mass effect were reported. The patient's past medical history was noncontributory. On physical examination, there was right-sided proptosis, and cranial nerves II-XII were grossly intact. The remainder of his neurologic examination was normal.

Subsequent magnetic resonance imaging (MRI) and computed tomography (CT) scans revealed a $4.6 \times 4.3 \times 3.4 \mathrm{~cm}$ transverse heterogenous enhancing mass centered at the right fovea ethmoidalis and lamina papyracea. This mass occupied a space in the anterior cranial fossa, superior right orbit, and right ethmoid sinuses (-Fig. 1).

The encroachment of the mass on the right orbit resulted in the displacement of the right superior and medial rectus muscles. Importantly, the mass was described as having a ground-glass central matrix with a lower density rim surrounding scalloped central lesions, characteristics commonly associated with FD of the skull. ${ }^{10}$

An endoscopic transnasal biopsy was then performed, and the pathology report confirmed the diagnosis of FD with a cementifying component. The patient was subsequently brought back for definitive, minimally disruptive endoscopic resection of the tumor via transnasal and transorbital approaches.

The transnasal approach was started first, with fiduciary endoscopic Stryker navigational protocol (Stryker Corp., Kalamazoo, Michigan, United States). Once the patient was prepared and draped, we proceeded with a 0-degree endoscope in the right nostril. The ethmoidal region of the skull base was skeletonized and exposed in a posterior fashion until the superior orbital lesion was identified.

Once the bony lesion was encountered, it was palpated with a Cottle and found to be very soft and pliable. The curette was used for most of the resection. The straight suction was used for delicate dissection laterally and superiorly near the skull base. The lesion was resected posteriorly to the dura, but the dura itself was not violated. Once all the tissue was removed from the tumor that could be reached transnasally, we began the transorbital approach to the tumor (-Fig. 2).

At this point, the remainder of the tumor had to be excised from the orbit, and a transnasal approach would not have 
A
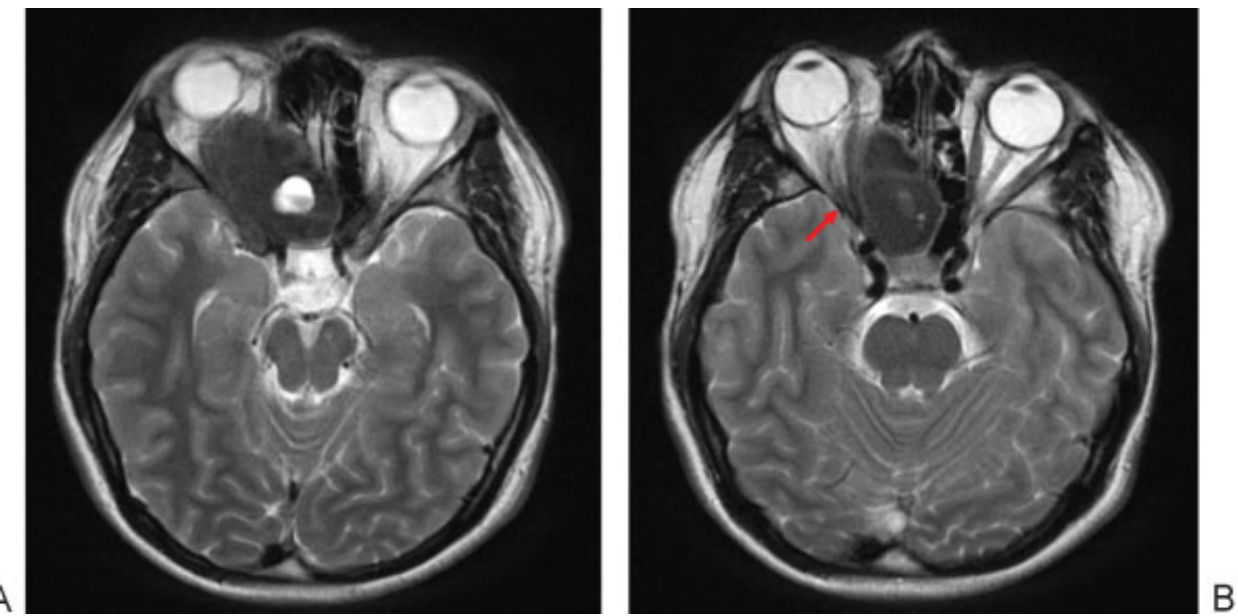

Fig. 1 Axial T2-weighted magnetic resonance images of the lesion. (A) The $4.6 \times 4.3 \times 3.4 \mathrm{~cm}$ bony lesion is centered on the right ethmoid roof and medial orbit. (B) There is mass effect upon underlying orbital structures, abutting the right superior orbit and slightly displacing the rectus muscles (arrow). Despite this, the patient's vision and ocular movements were normal. The optic nerve and ophthalmic vessels were not affected.

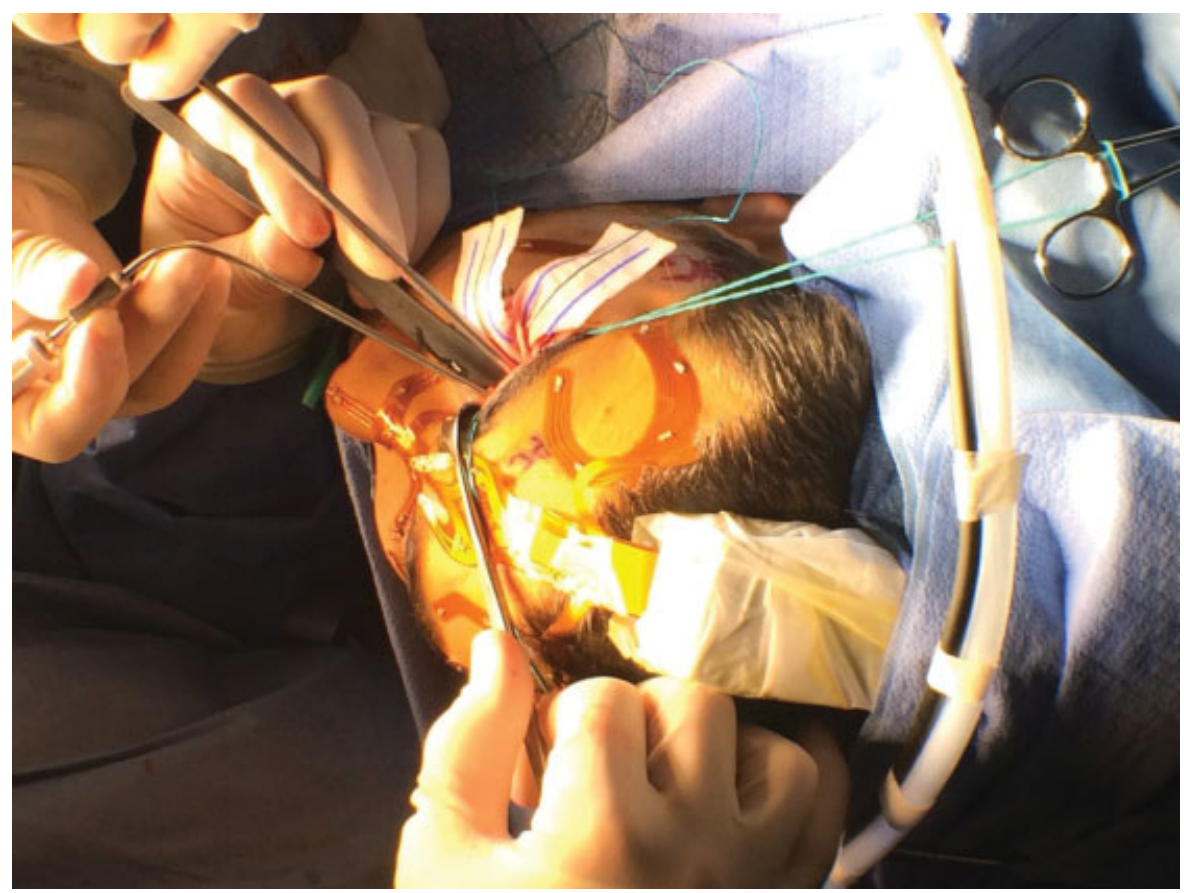

Fig. 2 Four-handed technique for transorbital endoscopic surgery.

provided adequate access. A superior transorbital approach was taken to access the rest of the tumor. A 2-cm transblepharoplasty incision was made in the right upper eyelid crease, via the superior eyelid crease approach, which is also described in a technical paper by Moe et al. ${ }^{6}$ This approach is very similar to that used for upper eyelid blepharoplasty; however, the plane of dissection continues in a preseptal plane between the periosteum and the orbital roof. One must take caution not to dissect into the fatty tissue deep to the orbital septum, which may violate the levator aponeurosis causing ptosis.

Once the superior orbital rim was identified, a Cottle was used to gently dissect tissue down to the level of the tumor.
Once this was located, a 0-degree endoscope was inserted. With the assistance of image guidance, the orbital aspect of this tumor was correlated to the location of our transnasal resection. A similar technique of curette and straight suction was used for delicate dissection of this tumor away from the orbital contents. Once all visualized portions of the FD tumor were resected through the transorbital technique, hemostasis was controlled, and a small amount of FloSeal (Baxter International Inc., Deerfield, Illinois, United States) was placed in the wound for additional hemostasis. The nasal passage was examined again for hemostasis or evidence of dural injury or CSF. 
The patient followed standard NYHNI protocols postoperatively for skull base surgery. Postoperative computed tomography (CT) scans showed removal of the bulk of the tumor with a small residual ground-glass bony involvement along the superolateral portion of the right orbital roof and along the perpendicular plate of the ethmoid bone. There was a partial resolution of the patient's orbital proptosis as evidenced by the postoperative CT scans and on physical examination. There were no postoperative visual, neurologic, or other complications. After 5 days, the patient was subsequently discharged well, with no residual pain or visual defects. However, there was a mild residual right-sided proptosis on discharge (-Fig. $\mathbf{3}$ ).

\section{Discussion}

FD is a benign osseous tumor in which fibrous and osseous tissue replaces normal medullary bone. It is an uncommon disease that comprises $2.5 \%$ of all bone tumors. ${ }^{11}$

Patients with FD of the cranium most often have frontal bone involvement, followed by sphenoid, ethmoid, parietal,
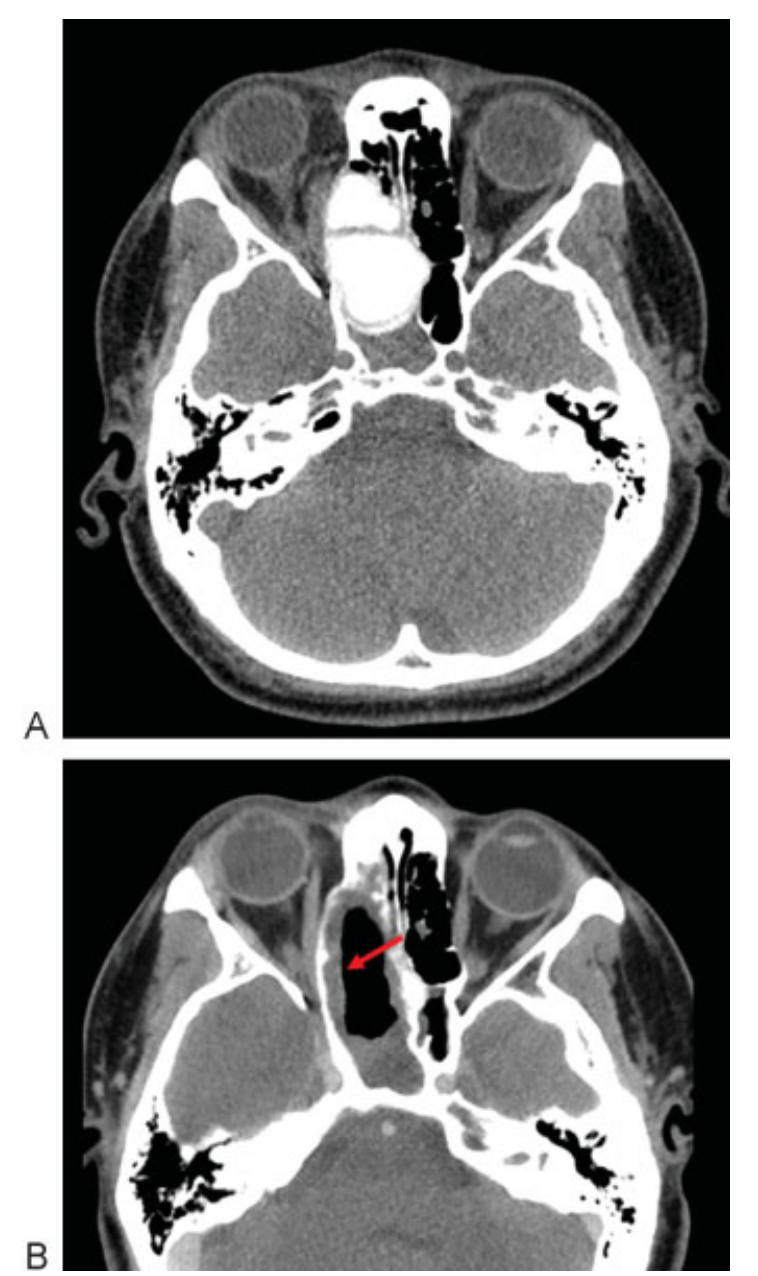

Fig. 3 (A) Preoperative axial computed tomography (CT) scan of the patient. (B) Postoperative axial CT scan of the patient. The bulk of the lesion has been resected, with improvement in the mass effect on orbital contacts (arrow). There remains a very mild relative right-sided proptosis. temporal, and occipital bone involvement. ${ }^{12}$ Furthermore, the skull base is the most common location of lesions in patients with FD of the cranium. Cranial FD symptoms usually include facial asymmetry and deformity, proptosis when the lesion involves the orbit, blurred vision, headache, and epiphora.

Surgery is the optimal treatment modality in FD treatment. According to previously published clinical criteria, FD necessitates surgical intervention when there is a chance that critical structures in the orbit may be compromised by mass effect. ${ }^{12}$ Our patient's FD was causing proptosis, and it was possible that the FD would increase in size and further compress the orbital contents. Therefore we decided to proceed with excising the tumor.

In this case, the tumor was in a location that would have been hard to access using uniportal access either via transnasal or transorbital routes. The CT scans previously described showed the tumor traversing the nasal cavity into the superior orbit as well as the anterior cranial fossa. A combined unilateral transnasal and transorbital approach would enable optimum approach vectors to access all these locations (-Fig. 4).

When transnasal instruments are coordinated in the region in the proximity of the sella, their working depth is $\sim 9 \mathrm{~cm}$ from the surface portal (the naris). ${ }^{5}$ At this depth, the boundaries of the orbital bones, nasal contents, and size of the piriform aperture cause a narrow funnel effect, limiting the working space. The working angle of bilateral transnasal instruments has been shown to be at a maximum of only 15 degrees when operating at or deep to the sella. ${ }^{13}$ This restricted angle often results in hand or instrument collisions, especially when multiple surgeons are operating simultaneously. Multiportal skull base surgery allows two surgeons to work with both hands at the same time, allows for the use of four instruments at once if needed together with navigation. This would overcome the limitation of endonasal procedures alone in which it is especially difficult for surgeons to manipulate their instruments within a narrow rigid corridor. Approach from two separate portals also allows surgeons to avoid critical structures that limit one approach.

In contrast to bilateral transnasal surgery, the working angles between instruments in combined transorbital and transnasal surgeries has been shown to be anywhere between 28 and 41 degrees. ${ }^{14}$ Combining transorbital/ transnasal portals provides a large range of approach vectors surgeons can choose, based on the location and characteristics of the lesion. Furthermore, instrument angles with respect to the sagittal plane and skull base plane are increased with combined portal modalities as compared with only transnasal or transorbital portals alone. Mean distances approaching a prechiasmatic target are also reduced in transorbital approaches. ${ }^{14}$ As described earlier, transnasal routes may have difficulty with access to pathologies at or near the optic nerves and chiasm, infundibulum, hypothalamic vessels, and the anterior cranial arteries. With combined multiportal techniques, lesions in these areas could potentially be reached safely. The 
A
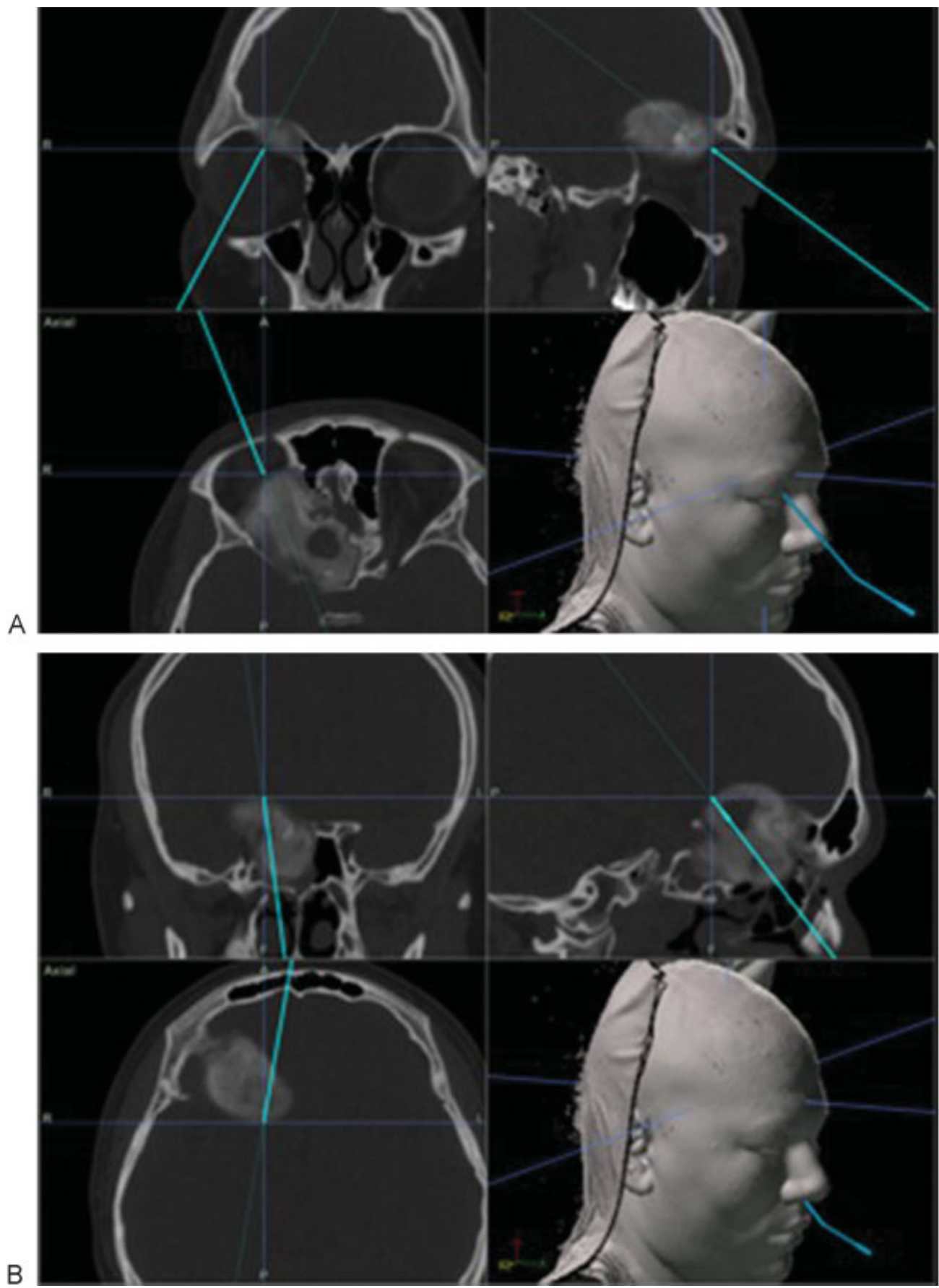

Fig. 4 (A) Navigational view of transorbital access into the anterior cranial fossa that offers the most direct route to the orbital and lateral portions of the fibrous dysplasia. (B) Navigational view of transnasal access. This access portal offers good access to the medial portions of the tumor but has limited access in reaching lesions that extend into the lateral portion of the anterior cranial base as well as lateral aspects of the middle and temporal cranial fossae. These images demonstrate how the limited approach vectors for each method may compensate for the other, increasing access to the pathology of interest.

superior aspects of these lesions are better visualized with transorbital or supraorbital approaches. ${ }^{6}$

With combined multiportal surgery, the surgical blind spots of either transnasal or transorbital surgery are reduced by compensating for the other method's approach angle restrictions. However, pathologies located either too posterior (past the squama temporalis) or in the lateral temporal fossa will be hard to access with either transnasal or trans- orbital methods. In such scenarios, another surgical route would be appropriate.

Some promising studies in computer models have used virtual endoscopy, which may help surgeons plan optimum portal locations depending on the patient's anatomy and location of the pathology. However, operator-based planning is still important to these systems, but it is conceivable that an algorithm-based program may soon come to the 
fore in planning endoscopic skull-based surgeries. Such systems are already being used in cardiothoracic and urologic surgeries, advising surgeons on optimum portal placements depending on the anatomy and site of pathology. $^{15,16}$

To date, only small case series or case reports have been published on multiportal endoscopic skull base surgery in live patients. To the best of our knowledge, there are at present only two reports on combined multiportal transnasal-transorbital approaches. ${ }^{17,18}$ Both of these studies involved the management of iatrogenic skull base defects. There is only one other recently published article that describes combined transnasaltransorbital endoscopic resection of neoplastic lesions. In that article, a case series of four patients with either malignant schwannoma or meningiomas have tumors excised, with no postoperative complications. ${ }^{19}$ It is because of this limited data on the combined transorbital transnasal method that we can only recommend this for benign lesions or simple nonneoplastic skull base defects.

\section{Conclusion}

Our case report presents a challenging tumor that surgeons at many institutions would have opted to perform with either open or uniportal transnasal/transorbital endoscopic surgery. The open procedures are associated with a higher morbidity and risk, and a longer postoperative stay. ${ }^{2}$ Single transnasal or transorbital methods to excise the tumor would have had trouble in accessing areas otherwise available to the combined portals.

This patient had a unique disease (FD) in a unique location that was treated without any postoperative complications. There is a paucity of literature describing combined endoscopic transnasal/transorbital access; to date only one other article has described the use of this technique in tumor excision. ${ }^{19}$

There is a need to compare this method against the traditional open approach/open with endoscopic approach/ or single portal endoscopic approaches. To date, simultaneous combined portal surgery has also not yet been performed. Because of the diverse patient population and pathologies that may require these interventions, it is inherently difficult to design a study that objectively compares these treatment modalities.

The success with endoscopic procedures over open procedures ostensibly favors the paradigm of combined endoscopic surgery, but further research is needed to establish this. In this case study, we showed how a single patient may be treated successfully with this surgical modality.

\section{Acknowledgments}

We would like to acknowledge the work of Maxine McGredy of Harvard University for her assistance in early drafts of this manuscript.

\section{References}

1 Janecka I. Skull Base Surgery. Vol 1. Philadelphia, PA: LippincottRaven; 1997

2 Dave SP, Bared A, Casiano RR. Surgical outcomes and safety of transnasal endoscopic resection for anterior skull tumors. Otolaryngol Head Neck Surg 2007;136(6):920-927

3 Kassam AB, Gardner P, Snyderman C, Mintz A, Carrau R. Expanded endonasal approach: fully endoscopic, completely transnasal approach to the middle third of the clivus, petrous bone, middle cranial fossa, and infratemporal fossa. Neurosurg Focus 2005; 19(1):E6

4 Aydin S, Cavallo LM, Messina A, et al. The endoscopic endonasal trans-sphenoidal approach to the sellar and suprasellar area. Anatomic study. J Neurosurg Sci 2007;51(3):129-138

5 Ciporen JN, Moe KS, Ramanathan D, et al. Multiportal endoscopic approaches to the central skull base: a cadaveric study. World Neurosurg 2010;73(6):705-712

6 Moe KS, Bergeron CM, Ellenbogen RG. Transorbital neuroendoscopic surgery. Neurosurgery 2010;67(3, Suppl Operative): ons16-ons28

7 Balakrishnan K, Moe KS. Applications and outcomes of orbital and transorbital endoscopic surgery. Otolaryngol Head Neck Surg 2011;144(5):815-820

8 Lim JH, Sardesai MG, Ferreira M Jr, Moe KS. Transorbital neuroendoscopic management of sinogenic complications involving the frontal sinus, orbit, and anterior cranial fossa. J Neurol Surg B Skull Base 2012;73(6):394-400

9 Chen HI, Bohman LE, Loevner LA, Lucas TH. Transorbital endoscopic amygdalohippocampectomy: a feasibility investigation. J Neurosurg 2014;120(6):1428-1436

10 Brown EW, Megerian CA, McKenna MJ, Weber A. Fibrous dysplasia of the temporal bone: imaging findings. AJR Am J Roentgenol 1995;164(3):679-682

11 Wei YT, Jiang S, Cen Y. Fibrous dysplasia of skull. J Craniofac Surg 2010;21(2):538-542

12 Amit M, Fliss DM, Gil Z. Fibrous dysplasia of the sphenoid and skull base. Otolaryngol Clin North Am 2011;44(4):891-902; vii-viii

13 Bly RA, Su D, Hannaford B, Ferreira M Jr, Moe KS. Computer modeled multiportal approaches to the skull base. J Neurol Surg B Skull Base 2012;73(6):415-423

14 Bly RA, Su D, Lendvay TS, et al. Multiportal robotic access to the anterior cranial fossa: a surgical and engineering feasibility study. Otolaryngol Head Neck Surg 2013;149(6): 940-946

15 Ogata M, Nagasaka M, Inuiya T, Makiyama K, Kubota Y. A development of surgical simulator for training of operative skills using patient-specific data. Stud Health Technol Inform 2011; 163:415-421

16 Bauernschmitt R, Feuerstein M, Traub J, Schirmbeck EU, Klinker G, Lange R. Optimal port placement and enhanced guidance in robotically assisted cardiac surgery. Surg Endosc 2007;21(4):684-687

17 Bly RA, Morton RP, Kim LJ, Moe KS. Tension pneumocephalus after endoscopic sinus surgery: a technical report of multiportal endoscopic skull base repair. Otolaryngol Head Neck Surg 2014;151(6): 1081-1083

18 Schaberg M, Murchison AP, Rosen MR, Evans JJ, Bilyk JR. Transorbital and transnasal endoscopic repair of a meningoencephalocele. Orbit 2011;30(5):221-225

19 Dallan I, Castelnuovo P, Locatelli D, et al. Multiportal combined transorbital transnasal endoscopic approach for the management of selected skull base lesions: preliminary experience. World Neurosurg 2015;84(1):97-107 